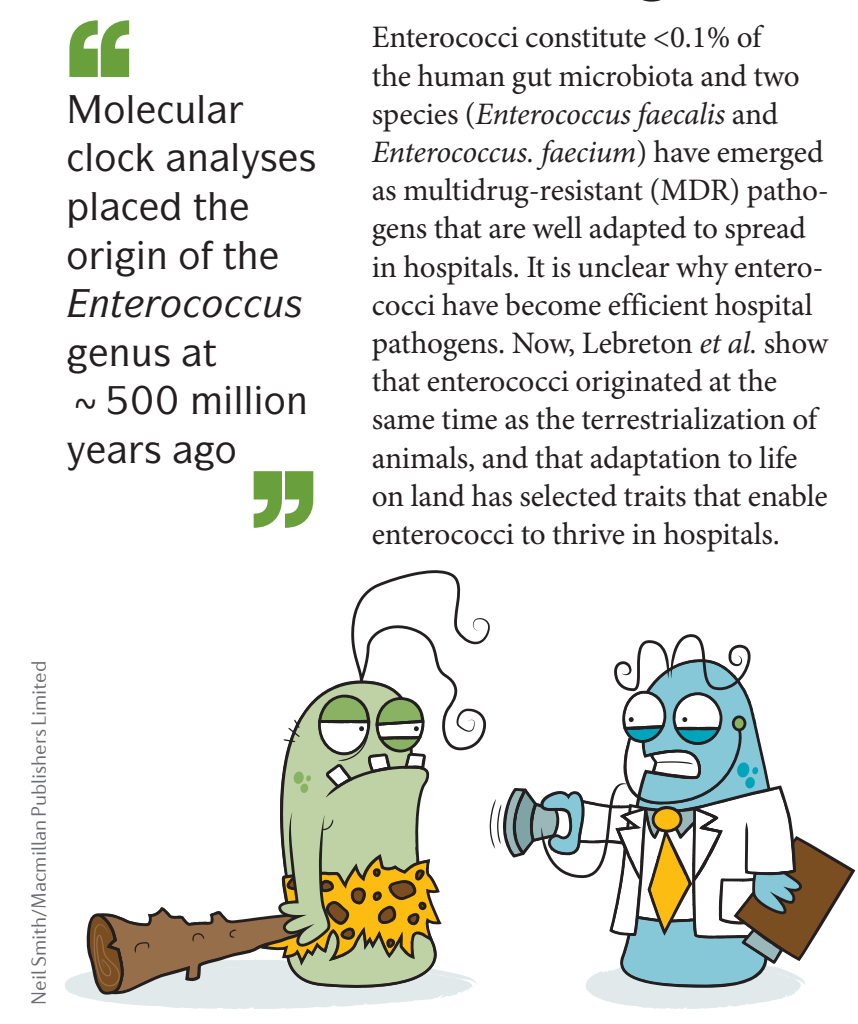

clock analyses

placed the origin of the

Enterococcus

genus at

500 million

\title{
The origins of pathogenic enterococci
}

To investigate the emergence of enterococci, the authors compared the sequences of 24 enterococcal species, five related species (including Vagococcus lutrae, which is usually isolated from marine hosts) and MDR pathogenic E. faecalis and E. faecium. Molecular clock analyses placed the origin of the Enterococcus genus at $~ 500$ million years ago (mya), which is consistent with the earliest fossil and molecular evidence of animal terrestrialization (425-510 mya). Furthermore, the authors mined reported gut microbiomes of animals and confirmed that enterococci were enriched in land life.

Sequence analyses were used to investigate the diversification of the Enterococcus genus. The authors identified 126 genes that were gained by enterococci since their divergence from the last common ancestor with $V$. lutrae. These genes were enriched in cell wall modification and stress response functions.
Furthermore, analyses of genes that were gained or lost during enterococci speciation showed that genes that were required for carbohydrate metabolism were under the greatest flux. This suggests that adaptation to various carbohydrates in the guts of new terrestrial animals may have driven speciation in enterococci. Next, the growth of enterococci and outgroup species was assessed under different conditions. Notably, all species of enterococci were resistant to environmental stresses, such as antibiotic exposure, disinfection, desiccation and starvation. This suggests that the adaptation to life on land has selected for certain traits that enable modern enterococci to become effective pathogens in hospitals.

Shimona Starling

ORIGINAL ARTICLE Lebreton, F. et al. Tracing the enterococci from Paleozoic origins to the hospital. Cell 169, 849-861.e13 (2017) 